UPR-671-T

hep-ph/9506386

June, 1995

\title{
Tunneling in quantum field theory with spontaneous symmetry breaking.
}

\author{
Alexander Kusenkol \\ Department of Physics and Astronomy \\ University of Pennsylvania \\ Philadelphia, PA 19104-6396
}

\begin{abstract}
Tunneling in quantum field theory is well understood in the case of a single scalar field. However, in theories with spontaneous symmetry breaking, one has to take into account the additional zero modes which appear due to the Goldstone phenomenon. We calculate the false vacuum decay rate in this case. The result may differ significantly from the tunneling rates in the absence of symmetry breaking.
\end{abstract}

\footnotetext{
* email address: sasha@langacker.hep.upenn.edu
} 
The effects of tunneling between different vacua in quantum field theory play an important role in high-energy physics, cosmology and condensed matter physics. The rate of the false vacuum decay was calculated in Refs. [1, 2, [3] for a potential which depends on a single scalar field. However, most theories beyond the Standard Model, e.g., the MSSM, grand unified theories, and strings, employ scalar potentials which depend on several scalar fields, and may have degenerate minima related by some internal symmetry. We will see that in this case the results of Refs. [1, 2, [3] do not apply because of the complications that arise due to the additional zero-modes associated with internal symmetries. The purpose of this letter is to generalize the semiclassical calculation of Refs. [2, 3] to such cases.

If the potential $U(\phi)$ depends on a single scalar field $\phi(x)$, then the corresponding path integral,

$$
\int[d \phi] e^{-S[\phi]}
$$

is dominated by the field configuration $\bar{\phi}(x)$ called the "bounce" and can be evaluated using the saddle point method [2, 3]. Then the transition rate per unit volume, in the semiclassical limit [3] is

$$
\Gamma / \mathrm{V}=\frac{1}{h^{2}}(S[\bar{\phi}])^{2} e^{-S[\bar{\phi}] / \hbar}\left|\frac{\operatorname{det}^{\prime}\left[-\partial_{\mu}^{2}+U^{\prime \prime}(\bar{\phi})\right]}{\operatorname{det}\left[-\partial_{\mu}^{2}+U^{\prime \prime}(0)\right]}\right|^{-1 / 2} \times(1+O(\hbar))
$$

where $S[\bar{\phi}]$ is the Euclidean action of the bounce, and $\operatorname{det}^{\prime}$ stands for the determinant with the four zero eigenvalues omitted. These four zero modes are related to the translational invariance in the four-dimensional Euclidean space. Integration over each of them yields a factor of $\sqrt{S[\bar{\phi}]}$ in equation (2)).

Let us now consider a quantum field theory with a scalar potential $U\left(\phi_{1}, \ldots, \phi_{n}\right)$ which has a local minimum at $\phi_{1}=\phi_{2}=\ldots=\phi_{n}=0, U(0, \ldots, 0)=0$ as well as at least one additional (local, or global) minimum at $\phi_{i}=\phi_{i}^{e}, i=1,2, \ldots, n ; U\left(\phi_{1}^{e}, \ldots, \phi_{n}^{e}\right)<0$.

The bounce, $\bar{\phi}(x)=\left(\bar{\phi}_{1}(x), \ldots, \bar{\phi}_{n}(x)\right)$, is the stationary point of the action, so that $\delta S[\bar{\phi}]=0$. It is found as an $\mathrm{O}(4)$-symmetric [4] solution $\bar{\phi}(r), r=\sqrt{x_{\mu} x^{\mu}}$, of the correspond- 


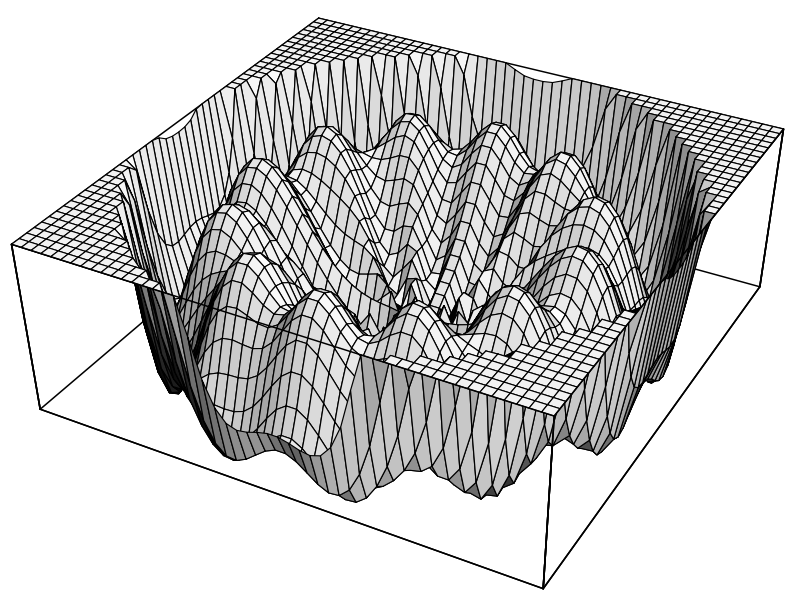

Figure 1: The potential $U\left(\phi_{1}, \phi_{2}\right)$ with several degenerate minima related by a $Z_{m}$ symmetry ing Euler-Lagrange equationst:

$$
\Delta \bar{\phi}_{i}(r)=\frac{\partial}{\partial \bar{\phi}_{i}} U\left(\bar{\phi}_{1}, \ldots, \bar{\phi}_{n}\right)
$$

with the following boundary conditions which ensure the finiteness of $S[\bar{\phi}]$ :

$$
\left\{\begin{array}{l}
\left.(d / d r) \bar{\phi}_{i}(r)\right|_{r=0}=0 \\
\bar{\phi}_{i}(\infty)=0
\end{array}\right.
$$

If the potential $U(\phi)$ has only one local minimum characterized by a negative value of the potential, equation (2) gives the correct answer for the rate of the false vacuum decay. Let us now consider the potential $U\left(\phi_{1}, \phi_{2}\right)$ which has $m$ degenerate local minima related by the $Z_{m}$

\footnotetext{
${ }^{1}$ In most cases, one cannot solve this system of non-linear equations analytically. An effective method for finding the bounce was proposed in Ref. [5].
} 
symmetry as shown in Fig. 1. The equation (3) will have $m$ independent solutions satisfying the boundary conditions (4), each bounce pointing in the direction of a given minimum in group space. Tunneling to different vacua are independent events, and therefore the total probability of the false vacuum decay is the sum of the contributions from each individual bounce. The naive application of the formula (2) might lead one to conclude that as the number of local minima $m$ increases, the lifetime of the false vacuum would approach zero. If one then considers the case of a broken $U(1)$ symmetry (Fig. 2) as the limit $m \rightarrow \infty$, one might argue that the false vacuum would decay infinitely fast because there are infinitely many channels, each having a finite probability determined by formula (2). This conclusion is wrong because, in the case of a continuous symmetry, the Goldstone modes give rise to some additional zero modes in the determinant in equation (2), so that the right-hand side of (2) is, in fact, divergent.

Clearly, formula (2) cannot be used to describe the tunneling probability in theories with spontaneously broken continuous symmetries. One must not include the Goldstone zero modes in the determinant, but deal with them instead in a similar manner to the way one usually treats the zero eigenvalues associated with translational invariance. The latter is the basic idea of our calculation.

We begin by considering the potential $U\left(\phi_{1}, \phi_{2}\right) \equiv U\left(\phi_{1}^{2}+\phi_{2}^{2}\right)$ which has a $U(1)$ symmetry (Fig. 2). We suppose that in addition to the local minimum at $\phi=0, U(\phi)$ has a $U(1)$ orbit of degenerate global minima at some non-zero value of $\phi$. It is convenient to redefine the fields in terms of the radial and the angular components:

$$
\left(\phi_{1}(x), \phi_{2}(x)\right)=\left(\eta(x) \operatorname{Re}\left(e^{i \theta(x)}\right), \eta(x) \operatorname{Im}\left(e^{i \theta(x)}\right)\right)
$$

Then $U(\phi)=U(\eta),(\partial / \partial \theta) U=0$, and every solution of the equation (3) can be written in the form

$$
\bar{\phi}(x)=e^{i \theta} \bar{\eta}(x)
$$




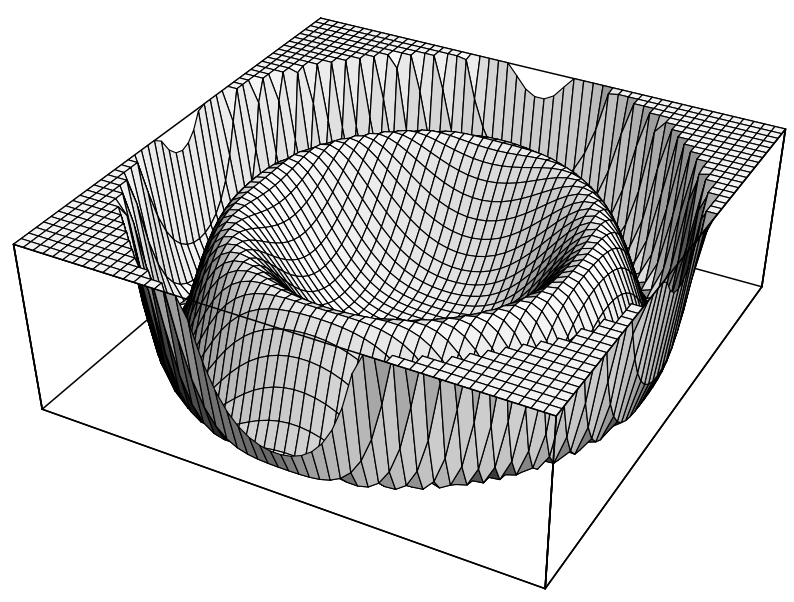

Figure 2: The potential $U\left(\phi_{1}, \phi_{2}\right) \equiv U\left(\phi_{1}^{2}+\phi_{2}^{2}\right)$ with a $\mathrm{U}(1)$ orbit of degenerate minima.

where $\theta$ is a constant independent of $x$ and $\bar{\eta}(x)$ is the usual bounce, the same as in the one-component case.

The idea of the method, used in Refs. [2, 3] to calculate the transition probability, was to evaluate the path integral using the saddle point method. The bounce $\bar{\phi}(x)$ is a stationary point of the action $(\delta S[\bar{\phi}]=0)$, but it is not a minimum because the second variation operator

$$
\frac{\delta S}{\delta \phi_{i} \delta \phi_{j}}=-\delta_{i j} \Delta+\frac{\partial^{2} U(\phi)}{\partial \phi_{i} \partial \phi_{j}}
$$

has one negative eigenvalue. In addition, this operator has four zero eigenvalues due to translational invariance in the coordinate space. The corresponding normalized eigenfunctions are [2, 3] 


$$
\phi_{\mu}=(S[\bar{\phi}])^{-1 / 2} \partial_{\mu} \bar{\phi}(x), \quad \mu=1, \ldots, 4
$$

In the vicinity of the bounce, one can expand the field $\phi(x)$ in the (complete orthonormal) basis of the eigenvectors of the operator (7):

$$
\phi_{k}(x)=\bar{\phi}_{k}(x)+\sum_{i} c_{k}^{(i)} \phi_{k}^{(i)}
$$

Then the path integral measure, $\left[d \phi_{k}\right]$, is defined as

$$
\left[d \phi_{k}\right]=\prod_{i}(2 \pi \hbar)^{-\frac{1}{2}} d c_{k}^{(i)}
$$

The path integration with respect to the Fourier coefficients $c_{k}^{(i)}$, which correspond to positive modes, is Gaussian and results in the determinant of the type (2) where the zero modes are not included. The integration over zero modes, however, is less straightforward. We would like to treat the Goldstone modes on the same footing as the Poincare zero modes (8). The first step is to find the orthonormalized basis in the subspace of eigenvectors that correspond to zero eigenvalues. By explicit differentiation of equation (3) with respect to $\theta$ we find that, as long as $U(\phi) \equiv U(\eta)$ is independent of $\theta$,

$$
\phi_{\theta}=\left(\int \bar{\eta}^{2}(x) d^{4} x\right)^{-1 / 2} \partial_{\theta} \bar{\phi}(x)
$$

is an eigenvector of (17) corresponding to a zero eigenvalue. The normalization comes from $\int\left|\partial_{\theta} \bar{\phi}(x)\right|^{2} d^{4} x=\int \bar{\eta}^{2}(x) d^{4} x$. It is easy to see that the eigenvector (11) is orthogonal to each of the eigenvectors (8). Therefore, the set of functions (8) and (11) combined form a complete orthonormal basis in the subspace of zero modes of the operator (7).

Let $c_{\theta}$ be the Fourier coefficient in (9) corresponding to the Goldstone mode. Then the change in $\phi$ corresponding to some small change in $c_{\theta}$ is $d \phi=\phi_{\theta} d c_{\theta}$. On the other hand, $d \phi=\partial_{\theta} \bar{\phi} d \theta$. Comparing these relations to each other, and using the normalization from equation (11), one gets 


$$
(2 \pi \hbar)^{-1 / 2} d c_{\theta}=\left(\int \bar{\eta}^{2}(x) d^{4} x / 2 \pi \hbar\right)^{1 / 2} d \theta
$$

Therefore, integration with respect to $c_{\theta}$ in the path integral (1) is equivalent to the integration over the angle $\theta$ in group space, up to the factor of $\sqrt{\int \bar{\eta}^{2}}$. The field configuration that comprises $n$ widely separated bounces is also a saddle point of the path integral with the action $n S[\bar{\phi}]$. We have to sum over all such configurations and integrate over the positions of the centers of the bounces. This is done in full analogy to the corresponding calculation in [2, [3].

The result is

$$
\Gamma / \mathrm{V}=2 \pi\left[\int \bar{\eta}^{2}(x) d^{4} x\right]^{1 / 2}\left(\frac{S[\bar{\eta}]}{2 \pi \hbar}\right)^{2} e^{-S[\bar{\eta}] / \hbar}\left|\frac{\operatorname{det}^{\prime \prime}\left[-\partial_{\mu}^{2}+U^{\prime \prime}(\bar{\eta})\right]}{\operatorname{det}\left[-\partial_{\mu}^{2}+U^{\prime \prime}(0)\right]}\right|^{-1 / 2} \times(1+O(\hbar))
$$

where det" stands for the determinant from which all zero eigenvalues, including the Goldstone zero mode, are excluded.

The result (13) can be easily generalized to the case of a larger internal symmetry group. Suppose the true vacuum is characterized by the vev that breaks some symmetry group $G$ down to $G^{\prime}$, so that the scalar potential has $N=\operatorname{rank}\left(G / G^{\prime}\right)$ Goldstone modes. The false vacuum decay rate is then

$$
\Gamma / \mathrm{V}=C\left(G / G^{\prime}\right)\left[\int \bar{\eta}^{2}(x) d^{4} x\right]^{N / 2}\left(\frac{S[\bar{\eta}]}{2 \pi \hbar}\right)^{2} e^{-S[\bar{\eta}] / \hbar}\left|\frac{\operatorname{det}^{\prime \prime}\left[-\partial_{\mu}^{2}+U^{\prime \prime}(\bar{\eta})\right]}{\operatorname{det}\left[-\partial_{\mu}^{2}+U^{\prime \prime}(0)\right]}\right|^{-1 / 2} \times(1+O(\hbar))
$$

Here $C\left(G / G^{\prime}\right)$ is a dimensionless factor that depends on the quotient group $G / G^{\prime}$. The pre-exponential factor in square brackets has dimension $N$ in length units, and the determinant in the numerator, with $4+N$ zero modes omitted, has dimension $2(4+N)$ in excess of that of the denominator. The power $(-1 / 2)$ reduces the latter to $-(4+N)$, so that the total dimension of the right-hand side is minus four, as it should be.

Some comments are in order. We note that the result (14) can differ significantly from that obtained by using the formula (2) and ignoring the symmetries of the potential. For 
example, let us consider the case of $G=S U(5) \rightarrow G^{\prime}=S U(3) \times S U(2) \times U(1)$. There are $N=12$ independent Goldstone modes in this case. In the thin wall approximation, one can express $\int \bar{\eta}^{2}$ in terms of $S[\bar{\eta}]$ times some dimensionful quantity. Then it can be shown that the answer obtained by applying formula (2) and ignoring the Goldstone modes would be off by a factor of $(S[\bar{\phi}])^{6}$. For $S[\bar{\phi}] \sim 10-100$, the difference would be as great as six to twelve orders of magnitude.

We also note that the increase in the number of degenerate minima $m$ (Fig. 1), does not necessarily imply an increase in $\Gamma$. In fact, to accommodate $m$ degenerate minima, $m \rightarrow \infty$, separated by the fixed height barriers, one has to allow the curvature (in the $\theta$-direction) at the bottom of each minimum to grow as $\sim m^{2}$. This corresponds to $\sim(1 / m)$ behavior of the determinant factor in equations (13) or (14). Now the total decay rate is $m$ times the contribution of each minimum, $\Gamma \sim \Gamma_{0} m(1 / m) \rightarrow$ const. This is the resolution of the paradox posed in the beginning.

It is straightforward to generalize formula (14) to the case of quantum field theory at finite temperature [6].

We have assumed that $G^{\prime} \subset G$, which is the case for many applications in particle physics. However, in some cases the true vacuum may, at some temperature, have a larger symmetry than the false vacuum (see, e.g., Ref. [0]), so that $G \subset G^{\prime}$. Finally, the true vacuum may be characterized by the symmetry group, $G^{\prime}$, completely unrelated to that of the false vacuum, $G$. If this is the case, then in general one has to modify the expression (14) to take into account the particular features of the potential.

To summarize, we have calculated the tunneling probability in quantum field theory with spontaneously broken local or global symmetries.

The author would like to thank P. Langacker for many stimulating discussions and helpful comments. We are also grateful to G. Segre and P. Steinhardt for useful conversations. This work was supported by the U. S. Department of Energy Contract No. DE-AC02-76-ERO3071. 


\section{References}

[1] M. B. Voloshin, I. Yu. Kobzarev and L. B. Okun', Yad. Fiz. 20, 1229 (1974) [Sov. J. Nucl. Phys. 20, 644 (1975)].

[2] S. Coleman, Phys. Rev. D15, 2929 (1977).

[3] C. G. Callan and S. Coleman, Phys. Rev. D16, 1762 (1977).

[4] S. Coleman, V. Glaser and A. Martin, Comm Math. Phys. 58, 211 (1978).

[5] A. Kusenko, University of Pennsylvania preprint UPR-656-T (hep-ph/9504418).

[6] A. D. Linde, Phys. Lett B70, 306 (1977); ibid B100, 37 (1981); Nucl. Phys. B216, 421 (1983).

[7] P. Langacker and S.-Y. Pi, Phys. Rev. Lett. 45, 1 (1980). 